Ophthalmology in Africa

\section{Minding the gap}

\section{A Harrison}

\section{The gap between ophthalmology in parts of Africa and more developed countries remains large, and ... is growing}

$\mathrm{T}$ ECWA Eye Hospital in Kano, Nigeria, is a mission hospital started by Dr Hursch, an American ophthalmologist, in the early 1940s. Although there are no surviving records from the time of Dr Hursch, I would venture to say that his practice of ophthalmology in Nigeria was not much different from his practice in America. Since the cutting edge tools of his day were loupes, and a small set of instruments, those items could easily be purchased, transported, and maintained in the setting of a developing country. Now, 60 years later, the situation is very different.

The eye hospital in Kano remains a bright spot in west Africa for surgery and treatment of eye diseases, and gives invaluable surgical experience to ophthalmologists in training. However, when I arrived in Kano in 2003, I expected that my practice of ophthalmology would be very different from what I was used to in America, and I was not disappointed.

Consider the differences.

Just a few years ago in America, I attended a seminar about improving outcomes of cataract surgery. One presenter boasted (rightfully so!) about his accurate selection of IOL (intraocular lens) power within plus or minus 0.25 dioptres, $100 \%$ of the time for a large series of patients. He did this by meticulous keratometry, immersion Ascans, and use of a third generation software package. In Africa, there are still many ophthalmologists struggling to convert to ECCE (extracapsular cataract extraction) surgery from ICCE (intracapsular cataract extraction). Many do not have operating microscopes, and IOLs are hard to come by because of cost and difficulties in importing. For those who do have access to operating microscopes and IOLs, a "standard" IOL power is most often used, since even fewer ophthalmologist have keratometers or A-scans. Couching of cataracts is still practised by traditional healers, even in large urban centres.

The treatment of glaucoma in developed countries is aided by an ever increasing armamentarium of medications, optic nerve head analysers, computerised visual field machines, seton implants, and antifibrotic agents. In Africa, most ophthalmologists' diagnostic tools are limited to Schiotz tonometers and direct ophthalmoscopes. Treatment for glaucoma is also simplified since timolol and pilocarpine are the only drugs readily available, so trabeculectomy is often done earlier. We will skip the comparisons for retina and refractive surgery where the disparities are even greater.

An important factor in this widening gap is the information explosion and rapid pace of technology in the more developed countries. If you are reading this, you likely have access to dozens of specialty and subspecialty journals. When looking back at how cataract surgery has changed just in the past 15 years, the differences are amazing.

Unfortunately, for ophthalmologists in the lesser developed countries, the ability to keep pace with the information explosion is hampered by a number of problems including the relatively high cost of journal subscriptions, and inadequate postal services. Those who do receive journals will find it difficult to implement the new technology and products because of the high costs, and the challenges of importation.

The gap is growing not simply because of the rapid pace of the more developed countries, but also because of myriad complicated problems in poorer countries. Widespread poverty, poor education systems, governmental corruption, AIDS, and inadequate medical training programmes, because of lack of resources and supervision, all contribute to "holding back" the pace of ophthalmic care.

Of course these generalisations do not always hold true. There are places where state of the art ophthalmology is practised in sub-Saharan Africa outside of South Africa, but those places are uncommon. For the majority of Africans, the ophthalmic care received is very different from what we are accustomed to in the more developed countries.

One interesting aspect of the gap is that it goes both ways. Not only are Africans affected by the gap, but ophthalmologists trained in the more developed countries who wish to practise in the less developed parts of Africa are also affected by it. I was fortunate that when I did my ophthalmology training I was taught standard ECCE as well as phacoemulsification surgery. However, currently, many ophthalmology training programmes in America teach only phacoemulsification surgery. If one of those newly trained ophthalmologists wished to practise in Africa, he or she would need additional training. This too may contribute to the widening gap as fewer ophthalmologists will be qualified to practise in developing countries, and fewer will be willing to take a "step backward" in their field.

When referring to the gap, I do not mean to imply that practice in Africa has all the negative aspects. There are several features of practice in the lesser developed parts of Africa that are superior to practice in developed countries. At my workplace in Africa I can practise ophthalmology without many of the bureaucratic headaches we have in more developed countries. Chart documentation can be brief and to the point with no worry about insurance documentation requirements, and much less concern about liability. We are forced to use only technology that is cost effective, and there is little pressure to adopt expensive new technologies with dubious clinical benefit.

There are many individuals, nongovernmental organisations, and international health organisations that are working to narrow the negative aspects of the gap. Pervasive availability and usage of the internet may narrow the gap in the information explosion. Low cost educational materials provided by the American Academy of Ophthalmology have been very helpful for training ophthalmologists overseas. "Twinning" is an excellent method whereby collaborations between institutes in developed countries and lesser developed countries occur. Such collaborations can be mutually beneficial, and lead to practice enhancements on both sides. ${ }^{1}$

However, the gap between ophthalmology in parts of Africa and more developed countries remains large, and in my opinion is growing. We must do more to narrow the gap, and to build bridges to our ophthalmology colleagues, and patients on the other side. There are positive and negative aspects to practice on both sides of the gap, and we would all do well to "mind the gap."

Br J Ophthalmol 2005;89:525. doi: 10.1136/bjo.2005.066001

Correspondence to: D A Harrison, ECWA Evangel Hospital, Zaria Bypass Road, Jos, Plateau State, Nigeria;

dllharrison@msn.com

\section{REFERENCE}

1 www.eye.usyd.edu.au/twinning/proposal/ executive.html. 
Trachoma

\section{How to assess the prevalence of trachoma}

\section{H R Wright, H Vu, H R Taylor}

\section{TRA for intervention in higher prevalence areas, ASTRA for low prevalence areas}

$\mathrm{T}$ achoma is the world's leading cause of infectious blindness, an estimated 84 million people have active trachoma and 7.6 million have trachomatous trichiasis. ${ }^{1}$ It is a disease of poor personal and community hygiene, affecting those living in the poorest conditions, and disappears as living conditions improve. Repeated or persistent infection with the obligate intracellular bacteria Chlamydia trachomatis results in the clinical syndrome of blinding trachoma. Trachoma progresses from inflammation of the upper tarsal conjunctiva to scarring; distortion of the eyelid causes trichiasis and eventual loss of vision secondary to corneal opacity after which blindness is essentially irreversible. The SAFE strategy developed by the World Health Organization (WHO) is effective in controlling blinding trachoma. ${ }^{2}$ It targets trachoma intervention at various stages of the cycle of disease: Surgery for trichiasis, Antibiotics for active trachoma, Facial cleanliness, and Environmental improvements. However, simple, reliable, and cost effective systems are needed to identify populations at risk of the blinding complications of trachoma and to assess the effectiveness of trachoma intervention programmes.

\section{METHODS TO ESTIMATE} TRACHOMA PREVALENCE

Population based prevalence surveys are the gold standard for estimating the prevalence of active trachoma and trachomatous trichiasis within a community. They have proved the mainstay of targeting and monitoring trachoma intervention; however, they are expensive, time consuming and may utilise resources that could be better spent on intervention programmes. The WHO has published guidelines advising how such a survey can be carried out in order to obtain a good random sample and provide accurate data. ${ }^{3}$ However, such surveys are relatively weak at distinguishing between a low level of trachoma and the absence of trachoma unless they have a very large sample size (figs 1 and 2). This is an important weakness in certifying that an area is free of disease.

Trachoma rapid assessment (TRA) developed by the WHO attempts to quickly, cheaply, and efficiently obtain the information needed to identify and prioritise areas for intervention programmes. ${ }^{4}$ It uses a two phase sampling technique to optimally bias the sample to the "worst places" within those communities most likely to have trachoma. At-risk communities are selected from within a region on the basis of existing trachoma information and known socioeconomic conditions. For this reason it can be confidently stated that if trachoma is not found in the "worst areas" it is most unlikely to be found anywhere else within that region. Unfortunately, some have tried to use data from TRA surveys to give prevalence estimates. TRA was explicitly designed not to yield prevalence data as it selects an "optimally biased" sample in order to detect trachoma if present.

Lot quality assurance sampling (LQAS) has also been trialled for the

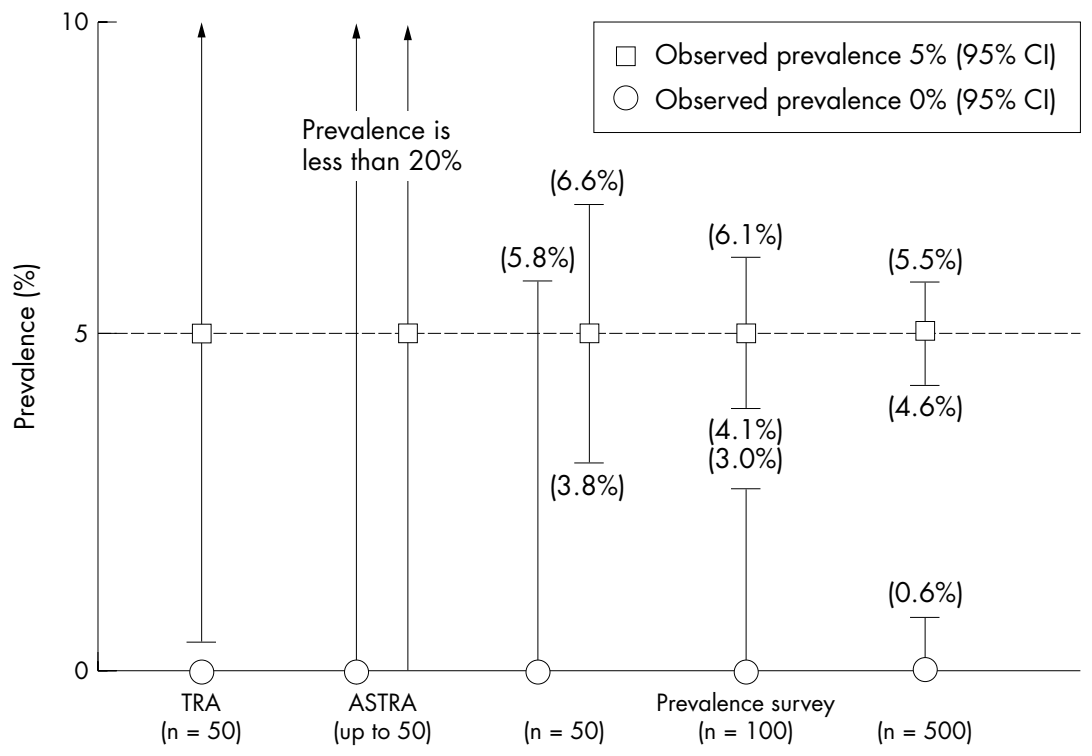

Figure 1 The 95\% confidence interval (Cl) for each survey method when the observed prevalence is $5 \%$ and $0 \%$. Circles/squares show the observed prevalence, bars show $95 \% \mathrm{Cl}$ limits (actual value in parentheses), and arrows signify that there is no limit in this direction for this survey type. as low or high prevalence and the values of these categories can be adjusted by selecting the cut-off point at which sampling stops.

\section{BRIEF SUMMARY OF FIELD TRIALS OF TRA AND ASTRA}

Results of several TRA field tests have been published some of which have compared TRA with prevalence survey results. ${ }^{6-9}$ They suggest that TRA is reasonably accurate in prioritising communities with higher levels of active trachoma. TRA did less well ranking communities with a low prevalence, although this is relatively less important as these communities were almost always assigned a low priority ranking. All studies reported that TRA was quicker and cheaper than a prevalence study. Two studies ${ }^{68}$ reported that there was an overemphasis on risk factor scores.

There is one published report of the effectiveness of ASTRA. ${ }^{5}$ The threshold was set at 14 and a maximum of 50

rapid assessment of trachoma. ${ }^{5}$ It has long been used in manufacturing and more recently by public health services, predominantly to evaluate service delivery particularly with respect to vaccination coverage. When used as a tool for the rapid assessment of trachoma prevalence the technique has been referred to as asymmetrical sampling trachoma rapid assessment (ASTRA). Children are examined until either a predetermined number of cases with active disease are identified (high prevalence) or a total of 50 children are sampled without the cut-off point being reached (low prevalence). Communities are categorised 


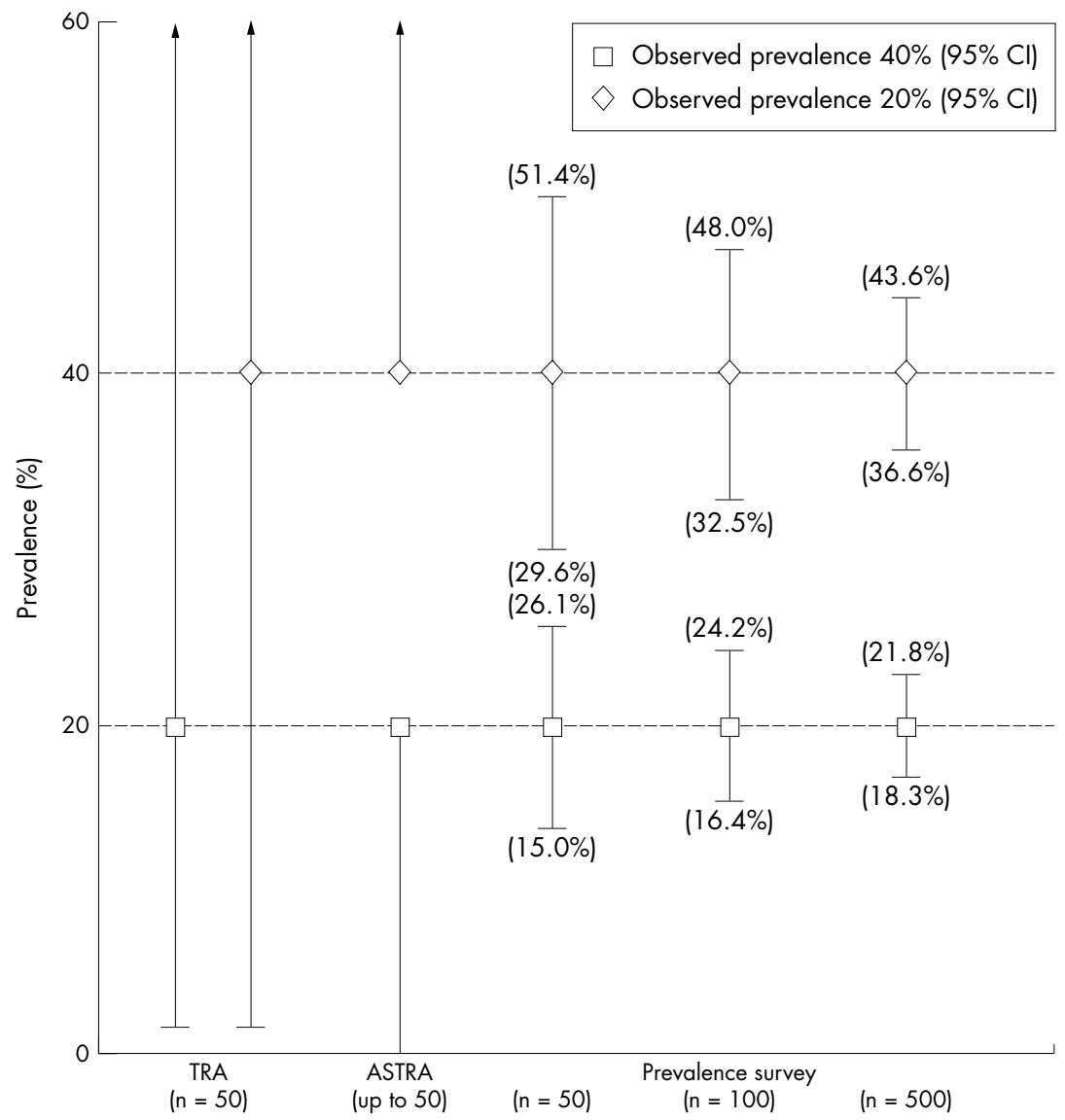

Figure 2 The $95 \%$ confidence interval (CI) for each survey method when the observed prevalence is $20 \%$ or $40 \%$. Squares/diamonds show the observed prevalence, bars show $95 \% \mathrm{Cl}$ limits, arrows signify that there is no limit in this direction for this survey type.

children aged 2-5 were identified for examination. They were able to accurately identify a community with a prevalence of $\leqslant 20 \%$ with a sensitivity of $94 \%$ and a prevalence of $\geqslant 40 \%$ with $95 \%$ sensitivity.

\section{WHICH METHOD IS MOST APPROPRIATE?}

According to the WHO guidelines a community should receive mass antibiotic treatment when the prevalence of active trachoma is more than $10 \%$ among 1-9 year old children. Treatment should continue for at least 3 years and should not stop until the prevalence is below 5\%.' Prevalence surveys remain the gold standard and are necessary to monitor intervention programmes, but TRA and ASTRA have an important role. Deciding which survey method to use must take into account the aim of the survey, the anticipated trachoma prevalence, and any important local concerns.
When assessing an area to determine if and where an intervention programme should be implemented TRA provides the quickest and surest way of ascertaining whether trachoma exists or not. It will also assist in prioritising or ranking communities or areas for intervention. ASTRA could also be used but it may miss pockets of disease.

To monitor the progress of an ongoing trachoma intervention programme, ASTRA can give a broad brush guide of the prevalence of disease. However, the selection of some "sentinel" communities for repeat prevalence assessment may be preferred by some.

To certify the elimination of trachoma one would again turn to the targeted TRA method or else use a prevalence survey with a very large sample size.

\section{CONCLUSION}

When deciding on which survey technique to use it is important to consider the aim of the survey. TRA can accurately and rapidly prioritise communities for intervention in higher prevalence areas; however, ASTRA may be better in low prevalence areas. Once a community has been identified for intervention a prevalence survey, or possibly ASTRA could be undertaken to allow programme monitoring. Finally, in order to certify that a region is clear of trachoma TRA is the most efficient method.

\section{ACKNOWLEDGEMENTS}

This research at the Vision Cooperative Research Centre was partly supported by the Australian Federal Government through the Cooperative Research Centres Program.

Br J Ophthalmol 2005;89:526-527. doi: $10.1136 /$ bjo.2005.066183

Authors' affiliations

H R Wright, H Vu, H R Taylor, Centre for Eye Research Australia (CERA), Vision CRC Victoria, Australia

Correspondence to: Dr Heathcote R Wright CERA, Locked Bag 8, East Melbourne, Vic 8002, Australia; h.wright2@pgrad.unimelb. edu.au

Competing interests: none.

\section{REFERENCES}

1 World Health Organization. Report of the 2nd global scientific meeting on trachoma, WHO/PBD/GET 03.1. Geneva: WHO 2003:10.

2 Kuper H, Solomon AW, Buchan J, et al. A critical review of the SAFE strategy for the prevention of blinding trachoma. Lancet Infect Dis 2003:3:372-81

3 World Health Organization. Primary health care level management of trachoma, WHO/PBL/ 93.33. Geneva: WHO, 1993

4 Negrel AD, Taylor HR, West S. Guidelines for the rapid assessment for blinding trachoma, WHO document WHO/PBD/GET/00.8. Geneva: World Health Organization and International Trachoma initiative, 2001.

5 Myatt M, Limburg $\mathrm{H}$, Minassian D, et al. Field trial of lot quality assurance sampling survey method for rapid assessment of prevalence of active trachoma. Bull World Health Organ 2003;81:877-85.

6 Limburg H, Bah M, Johnson GJ. Trial of the trachoma rapid assessment methodology in the Gambia. Ophthalmic Epidemiol 2001;8:73-85

7 Paxton A. Rapid assessment of trachoma prevalence-Singida, Tanzania. A study to compare assessment methods. Ophthalmic Epidemiol 2001;8:87-96.

8 Liu H, Ou B, Paxton A, et al. Rapid assessment of trachoma in Hainan provence, China: validation of the new World Health Organization methodology. Ophthalmic Epidemiol 2002;9:97-104.

9 Rabiu MA, Alhassan MB, Abiose A. Trial of trachoma rapid assessment in a subdistrict of northern Nigeria. Ophthalmic Epidemiol $2001 ; 8: 263-73$ 